\title{
EBSD Multiphase Preparation: U-10Mo, Zr, Al
}

\author{
Carl Necker, Ann Kelly
}

Los Alamos National Laboratory, Material Science and Technology: Metallurgy, MS: G770, Los Alamos, NM 87545

The preparation of multiphase surfaces for Electron Backscatter Diffraction (EBSD) often requires different techniques to yield indexable patterns. The crux of this study was to establish preparation techniques that yielded successful scans on each of the phases. The multiphase system was a hot isostatic bonded composite of a central rolled plate of U-10 Mo, a thin interlayer of $\mathrm{Zr}$ and an outer plate of rolled $6061 \mathrm{Al}$. The mounted cross-sections were ground and polished using normal metallographic procedures followed by electropolishing with material specific solutions to evaluate the uranium alloy and the aluminum. An agitated perchloric solution, chilled to $-20^{\circ} \mathrm{C}$, electropolished the aluminum surfaces, providing easily indexed patterns on the aluminum. No patterns were achieved on the $\mathrm{Zr}$ or $\mathrm{U}$ alloy using this solution. An agitated, room temperature phosphoric acid solution was used to electropolish the uranium alloy. Initial attempts yielded less than stellar results on the uranium due to galvanic coupling between the aluminum and uranium alloy. This issue was overcome by masking the aluminum features, yielding clear, easily indexed U alloy patterns. An additional customer requirement was the development of a preparation technique that does not require etchants or electropolishing solutions. Initial mechanical polishing, finished with a colloidal silica prep, yielded indexable patterns from the uranium alloy, aluminum as well as the zirconium. Figure 1 compares $U$ alloy patterns after electropolishing and following only mechanical polishing. As one might expect, electropolishing removes the mechanical polish disturbed surface layer, yielding significantly improved patterns. The weaker patterns were clear enough for scan recognition however the scan times were significantly longer to allow for improved pattern recognition. Figure 2 shows the inverse pole figure maps resulting from an 'electropolished' scan and a 'mechanical polished only' scan. Grain boundaries were not drawn in the polish-only scan due to the significant amount of orientation variation inside individual grains. Equivalent material was used for both scans, material that was fully recrystallized so there should be no intragranular misorientations. The electropolished scan shows no intragranular misorientations whereas the as-polished scan does. These misorientations are an artifact of the preparation process. However, the intragranular misorientations do not result in artificial grains as the misorientations are normally $2^{\circ}-5^{\circ}$ therefore the overall grain size calculations are mostly unaffected. The texture is mostly unaffect when the low confidence index points (artificial) are ignored. The 6061 aluminum showed very similar results with weaker patterns and artificial low angle intragranular misorientations. Figure 3 compares the distribution of confidence indices for the electropolished and as-polished scans. A significantly higher fraction of very low confidence points, less than 0.05 , exist in the as-polished condition. Future work will endeavor to improve upon the as-polished conditions either by decreasing the incremental size steps of the polishing media, increasing the length of time of polishing steps as well as determining if other existing polishing media may provide a weak chemical polishing condition. 

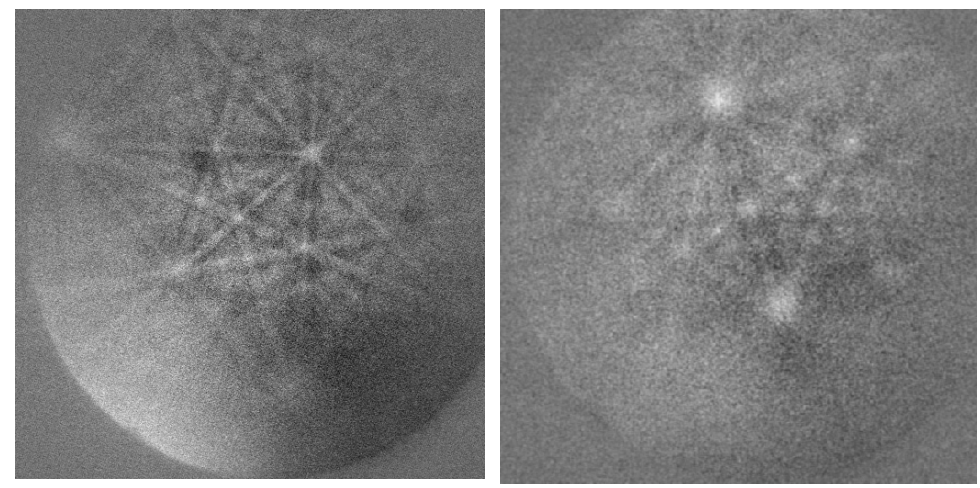

Figure 1. Left - U-10Mo pattern after electropolished finish. Right - U-10Mo pattern in mechanical as-polished condition.
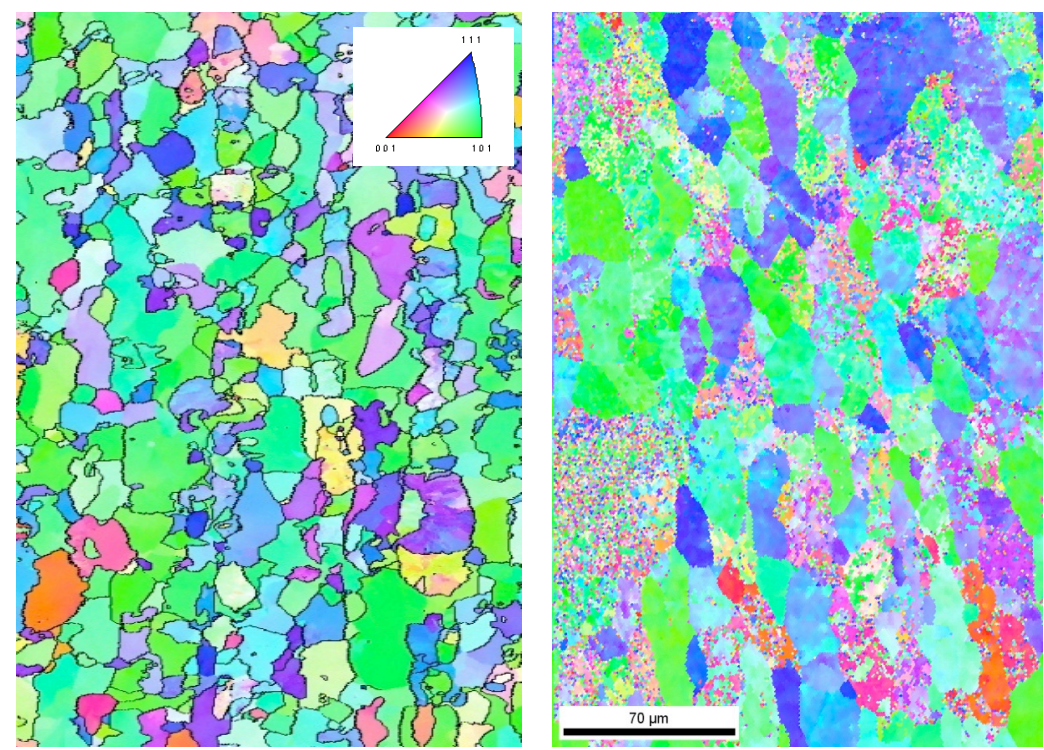

Figure 2. Left - U-10Mo scan after electropolishing. Right - U-10Mo scan as-polished.

Cenddence Inder

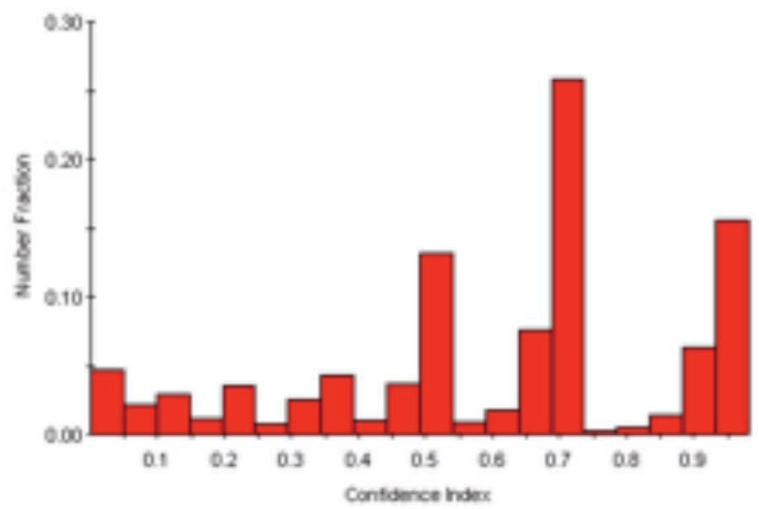

Cendsence Indes

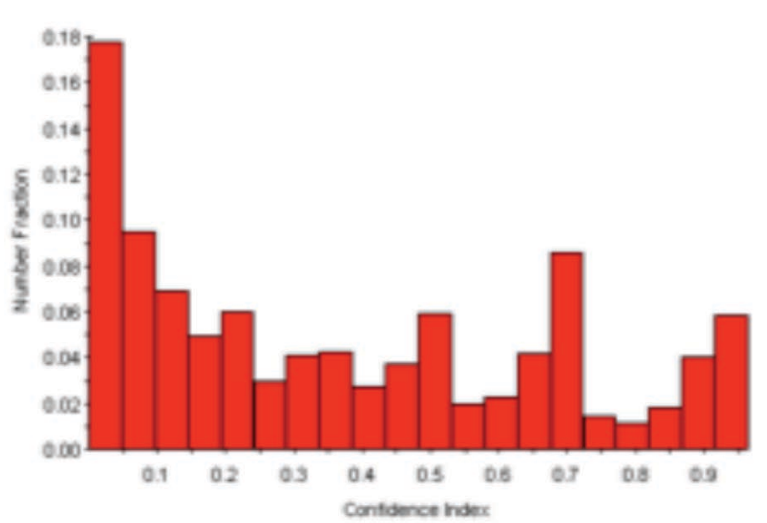

Figure 3. Confidence Index distributions. Left - 6061 Al after electropolishing. Right - $6061 \mathrm{Al}$ after mechanical polishing. 